\title{
Comparison of the mass preconditioned HMC and the DD-HMC algorithm for two-flavour QCD
}

\section{Marina Marinkovic*}

Humboldt Universität zu Berlin, Institut für Physik, Newtonstr. 15, 12489 Berlin, Germany

E-mail: mmarina@physik.hu-berlin.de

\section{Stefan Schaefer}

Humboldt Universität zu Berlin, Institut für Physik, Newtonstr. 15, 12489 Berlin, Germany CERN, Physics Department, 1211 Geneva 23, Switzerland

E-mail: stefan.schaeferdcern.ch

Mass preconditioned HMC and DD-HMC are among the most popular algorithms to simulate Wilson fermions. We present a comparison of the performance of the two algorithms for realistic quark masses and lattice sizes. In particular, we use the locally deflated solver of the DD-HMC environment also for the mass preconditioned simulations.

The XXVIII International Symposium on Lattice Field Theory, Lattice2010

June 14-19, 2010

Villasimius, Italy

${ }^{*}$ Speaker. 


\section{Introduction}

In order to accelerate numerical simulations in lattice QCD, different preconditioning techniques are being used. We will try to make a comparison between two popular ways of preconditioning the Hybrid Monte Carlo (HMC) for improved Wilson fermions: domain decomposition introduced by Lüscher in the DD-HMC algorithm[1] and Hasenbusch's mass preconditioning (MP)[2]. In both approaches, the quark determinant is factorized into a part which is dominated by the infra-red and another which is largely ultra-violet. This leads to the reduction of the quark force magnitude in the molecular dynamics equations. Therefore, the associated integration step sizes can be set to larger values, which gives an acceleration of the algorithm.

Comparisons between algorithms are difficult. In a modern lattice QCD computation, many parameters have to be set. Since their number can go into the dozens-their optimal values depending on each other-it is virtually impossible to find the minimum, at which each algorithm performs best, and then make a true comparison. In particular, the performance is determined by the auto-correlation times of the observables of interest, whose measurements require runs with high statistics. Furthermore, the optimal values of the parameters and the relative performance of the algorithms might also depend on the implementation and the computer the simulation is run on.

At least from the point of view of implementation, we tried to have the comparison on virtually equal grounds: for the DD-HMC, we used Lüscher's publicly available code ${ }^{1}$ and from it, we developed an implementation of the mass preconditioned HMC, reusing as many building blocks as possible. In particular the locally deflated solver introduced in the DD-HMC framework[3] turns out to greatly speed up the simulations in both algorithmic setups.

The block decomposition, on which the DD-HMC is based, allows for a decoupling of the blocks for a large part of the forces in such a simulation. The links on the boundary are not updated during the trajectory, which results in reduced communication, and is therefore suitable for clusters with fast nodes and a relatively slow connection. However, this also means that only a fraction $R$ of the links is "active". The naive expectation that the auto-correlation times grow proportional to $R^{-1}$ is confirmed in pure gauge theory[4], however, with dynamical fermions the cost is not reduced accordingly. There is a competition between the need of the computer, which are small blocks using many processors of massively parallel machines, and the need of the physics, which asks for blocks of a physical size of at least $0.5 \mathrm{fm}$ to provide an efficient preconditioning. The size of the blocks will determine the relative size of the block force and the global remainder.

In mass preconditioning, the fermion matrix is preconditioned (basically) by one with a fermion of a larger mass. The value of its mass plays the role of the size of the block in domain decomposition, but it is continuous and therefore can be tuned. Also more than one preconditioning fermion can be easily used.

Both algorithms have been compared in [5], however, new to the present study is the use of the deflated solver in both algorithms. The DD-HMC is well documented in the literature, so we will only describe our setup for the MP-HMC algorithm in Section 2 and 3, and continue to our test in Sect. 4,5 and 6.

\footnotetext{
${ }^{1}$ http://cern.ch/luscher/dd-hmc
} 


\section{Action and algorithms}

We are simulating $N_{f}=2$ degenerate flavours of non-perturbatively $\mathrm{O}(a)$ improved Wilson quarks, using the Wilson gauge action. The Dirac operator in this formulation is given by

$$
D(m)=D_{W}+c_{s w} \sum_{\mu, v} \frac{i}{4} \sigma_{\mu v} \hat{F}_{\mu v}+m,
$$

where $D_{W}$ represents the unimproved Wilson Dirac operator without mass term, $c_{s w}$ is the improvement coefficient and $m$ the bare quark mass.

In our implementation of MP-HMC, we use mass preconditioning for the Schur complement of the symmetric even-odd preconditioning. Starting from the standard decomposition,

$$
\operatorname{det} Q=\operatorname{det}\left(\gamma_{5} D\right)=\operatorname{det} Q_{e e} \operatorname{det} Q_{o o} \operatorname{det} Q_{S} \quad \text { with } \quad Q_{S}=1-Q_{e e}^{-1} Q_{e o} Q_{o o}^{-1} Q_{o e},
$$

we write

$$
\operatorname{det} Q=\operatorname{det} Q_{e e} \operatorname{det} Q_{o o} \operatorname{det}[W(\Delta m)] \operatorname{det}\left[W^{-1}(\Delta m) Q_{S}\right]
$$

where $W(\Delta m)=Q_{S}+\Delta m$ with $\Delta m>0$. This leads to the effective action

$$
S_{\text {eff }}=-2\left(\log \operatorname{det} Q_{e e}+\log \operatorname{det} Q_{o o}\right)+\left|W^{-1}(\Delta m) \Phi_{1}\right|^{2}+\left|\left(1+\frac{\Delta m}{Q_{S}}\right) \Phi_{2}\right|^{2} .
$$

Using again a Schur complement approach, the inverse of the preconditioned operator $Q_{S}$ can be constructed from the inverse of the full Hermitian Dirac operator $Q$

$$
Q_{S}^{-1}=P_{e} Q^{-1} Q_{e e} P_{e}
$$

In the following, the forces associated with pseudo-fermion field $\Phi_{1}$ are denoted by $F_{1}$, whereas $F_{2}$ are the forces from $\Phi_{2}$.

In the DD-HMC, the quark determinant is written as the product of the determinants of the Dirac operator restricted to the blocks and a factor which accounts for the remaining contributions to the fermion determinant. The latter factor couples the gauge fields on the different blocks. The block forces are referred to as $F_{1}$ and $F_{2}$ is the block interaction force. For details of this setup see [1].

In both setups, UV/IR separation due to the preconditioning opens the possibility to integrate $F_{2}$ on a larger time scale than $F_{1}$.

\section{Simulation parameters}

We have performed runs on a $48 \times 24^{3}$ lattice at $\beta=5.3$ and $c_{s w}=1.90952$, corresponding to the lattice spacing in physical units of $a \approx 0.071 \mathrm{fm}$ from $r_{0}=0.5 \mathrm{fm} \mathrm{[6].} \mathrm{The} \mathrm{hopping} \mathrm{parameter}$ $\kappa_{\text {sea }}=0.13625$ corresponds to a pion mass of $m_{\pi} \approx 420 \mathrm{MeV}$ and $m_{\pi} L \approx 3.6$. In all our runs, the trajectory length is set to $\tau=0.5$, for which we take the normalization of Ref. [1]. These parameters were also used in DD-HMC simulations without deflation in Ref. [7].

In DD-HMC, the locally deflated Schwarz-preconditioned generalized conjugate residual (GCR) solver described in [3] is employed for the inversions in $F_{2}$. The less expensive inversions on the blocks, needed in the computation of $F_{1}$, are done with the BiCGstab algorithm. In the multiple 


\begin{tabular}{|c|c|c|}
\hline & without defl. & with defl. \\
\hline \hline$\left\langle N_{\mathrm{GCR}}\left(F_{2}\right)\right\rangle$ & 107 & 23 \\
\hline comp. time $\left(F_{2}\right)$ & $780.42 \mathrm{~s}$ & $264.98 \mathrm{~s}$ \\
\hline
\end{tabular}

Table 1: Average number of GCR iterations per trajectory and the total execution time for the $F_{2}$ computation in MP-HMC with the deflated solver and without the application of deflation. The size of the deflation blocks is $6^{2} \times 4^{2}$. Note that a reduction in average time by a factor $\sim 3$ is achieved, taking into account the time needed for the construction of the deflation subspace.

time scale integration we use $N_{1}$ steps in the fermion force $F_{1}$ for each of the $N_{2}$ steps per trajectory of $F_{2}$ and analogously $N_{0}$ steps of the gauge force per step in $F_{1}$. We choose $N_{2}, N_{1}$ and $N_{0}$ to be 18,5 and 6 , respectively, for a block size of $6^{2} \times 12^{2}$.

Without much tuning, for the MP case we have taken the same step size at the outer force and the rest of the parameters is chosen according to the ratio of the forces magnitude, i.e. $\left\|F_{2}\right\|$ : $\left\|F_{1}\right\|:\left\|F_{0}\right\|=1: 9: 36$. To be on the safe side, we have chosen $N_{2}, N_{1}, N_{0}=18,10,10$. In our version of MP-HMC, the Schwarz-preconditioned GCR solver is employed for the computation of both $F_{1}$ and $F_{2}$. The demanding inversions with the low quark mass in the $F_{2}$ computation are done with the deflated version of the solver whereas in the $F_{1}$ computations the deflation was off. Here, the preconditioning parameter is the positive mass difference added to the symmetrically preconditioned Dirac operator which we set to $\Delta m \approx 0.09$. More tuning could probably lead to a better performance than the one discussed below.

In both setups, the standard leap frog integration is implemented and for the prediction of the solution in all inversions, the chronological inversion method of Brower et al. [8] is used.

\section{Solver performance}

The Schwarz-preconditioned GCR solver used for all the inversions in MP-HMC is taken over from the DD-HMC environment. The results of intensive tests of this solver implementation within DD-HMC can be found in $[9,10]$. As expected, we find the improved performance of the deflated solver also in the MP-HMC, gaining roughly a factor of three in the time needed for the computation of $F_{2}$ on our lattices compared to the case without the application of deflation, details can be found in Table 1.

For the update of the deflation subspace the same criteria as in the DD-HMC setup were applied[10], however, since in MP-HMC all links are active, the deflation subspace has to be updated more often than in the DD-HMC case to satisfy the same update criteria, see Fig. 1. In principle, we could have optimized the update criterion of the deflation space even further to match the particular conditions in the MP-HMC given by the cost of the $F_{2}$ force computation, but we have already achieved a very significant gain and do not expect any further improvements to dramatically change the situation.

\section{Quark forces and stability}

At small quark masses, instabilities in the numerical integration of the molecular dynamics 

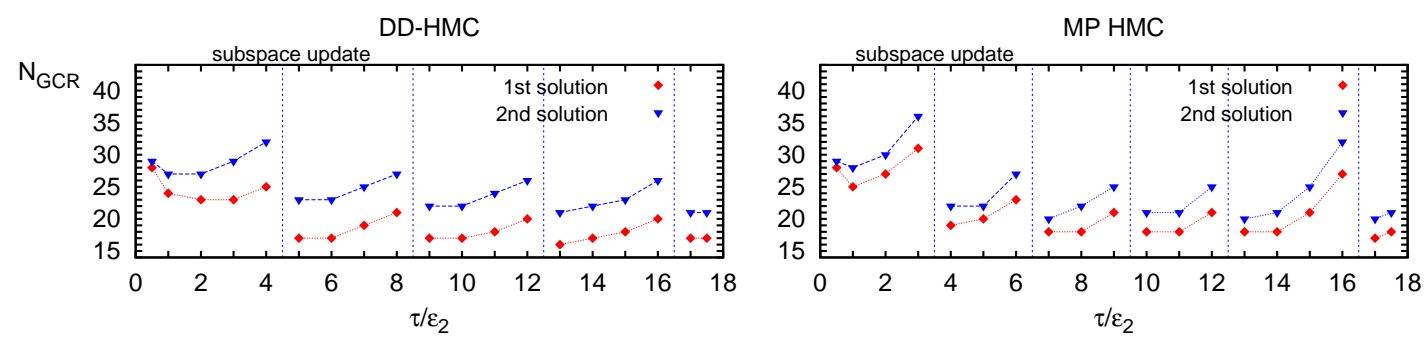

Figure 1: History of the iteration numbers $N_{\mathrm{GCR}}$ of the deflated Schwarz-preconditioned GCR solver along a molecular-dynamics trajectory, using the DD-HMC (left side) and MP-HMC (right side) algorithm, plotted against the molecular-dynamics time $\tau$ given in units of the integration step size $\varepsilon_{2}=\frac{\tau}{N 2}$. The lattice size in both cases is $48 \times 24^{3}$ and $\kappa_{\text {sea }}=0.13625$. The vertical lines indicate the refreshment of the deflation subspace.

equations may occur, which manifest themselves in violent fluctuations in the energy violation $\Delta H$ of the molecular dynamics evolution. According to the tests of the DD-HMC algorithm performed so far, severe integration instabilities were rare [7]. This has to be demonstrated also for the MP$\mathrm{HMC}$ and for both algorithms when going to smaller quark masses.

Typically, the force $F_{2}$ is the source of these instabilities, and in Fig. 2 we show Monte Carlo time histories for $F_{1}$ and $F_{2}$ showing the maximal and average forces, together with the corresponding $\Delta H$ throughout roughly 600 trajectories for DD-HMC and 300 in the MP-HMC case. One can see that the average force in the case of MP-HMC fluctuates a lot less than in DD-HMC, which is reflected in smaller magnitude and fluctuations in $\Delta H$ for the mass preconditioning.

\section{Efficiency of the algorithms}

The question of performance of the two algorithms must be addressed empirically and although a final answer would require to include in the comparison a series of lattice sizes and quark masses, our study could give us a first insight into how the two approaches relate in computational cost. This includes not only the cost of performing a single trajectory, but also the related auto-correlation times, because what matters is the cost of achieving a certain statistical error. The presented computations are performed on the SGI Altix which is built of Intel Xeon Gainestown X5570 CPUs with InfiniBand connections at HLRN supercomputing system at ZIB in Berlin and RRZN in Hannover, and the relative timings can certainly be different on other architectures.

In Table 2 we show the average plaquette value in both runs, integrated autocorrelation time $\tau_{\text {int }}$ and the acceptance rate for the two cases. As we have already mentioned, the fraction of active links in the DD-HMC for the chosen parameters is $R=36 \%$, increasing it would mean to run with fewer than 32 MPI processes. Note, however, that this is partially due to the relatively small $L / a=24$. It has been shown for the pure gauge theory that the autocorrelation time scales with the inverse of this fraction[4] as long as the blocks are of a reasonable size. In order to be able to compare the two algorithms, we have multiplied the current result for the integrated autocorrelation time with $R$, and scaled the execution time accordingly. Since the available statistics is not enough for the reliable estimation of the errors in the autocorrelation times, the values for the $\tau_{\text {int }}$ and its 


\begin{tabular}{|c|c|c|c|c|c|}
\hline & wall clock / R & $U_{P}$ & $\tau_{\text {int }}\left(U_{P}\right) \times R$ & $N_{\text {traj }}$ & acc. rate \\
\hline \hline DD-HMC & $2010 \mathrm{~s}$ & $1.65106(10)$ & $10(5)$ & 840 & $90(1) \%$ \\
\hline MP-HMC & $1530 \mathrm{~s}$ & $1.65127(10)$ & $10(4)$ & 432 & $85(2) \%$ \\
\hline
\end{tabular}

Table 2: Comparison of the DD-HMC algorithm with the MP-HMC. Both simulations are done for the improved Wilson theory with two degenerate fermion flavours. The lattice size is $48 \times 24^{3}$, lattice spacing $a \approx 0.07 \mathrm{fm}$ and the pion mass $m_{\pi} \approx 400 \mathrm{MeV}$. The block size in DD-HMC is $6^{2} \times 12^{2}$, while in the MP case, the difference in mass $\Delta m \sim 0.09$. Here $R$ represents the fraction of active links in the algorithm, $R=0.36$ for DD-HMC and $R=1$ for MP-HMC. The two algorithm demonstrate comparable performance.

error should be taken only as first estimates. Including the acceptance into consideration, we can conclude from the performed study that in both approaches roughly the same CPU time is needed for the same error in the measured observable $U_{P}$.

\section{Summary}

In this contribution, we have compared DD-HMC, one of the most efficient algorithms for
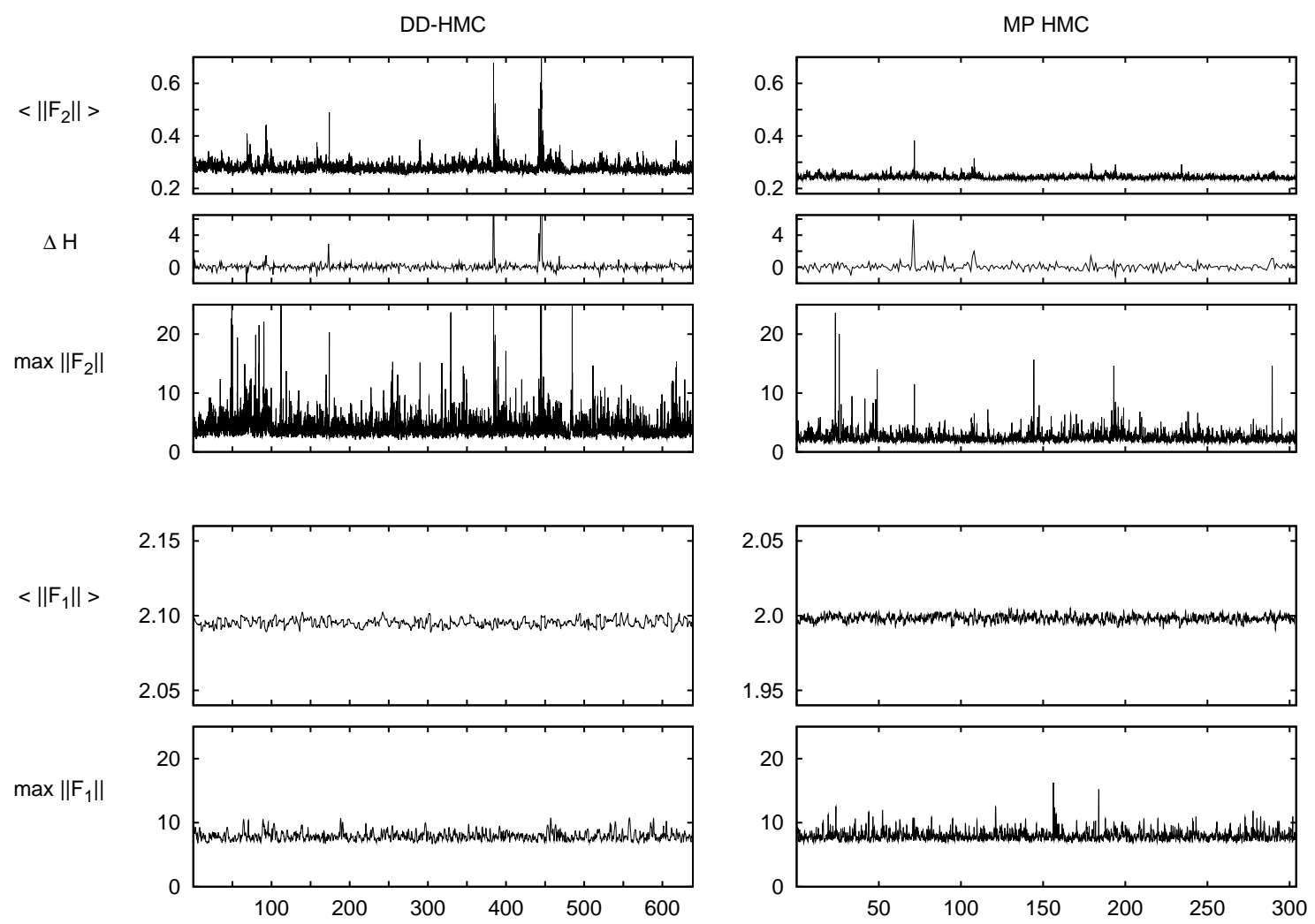

Figure 2: Histories of the energy violation $\Delta H$, as well as maximum and average forces $F_{2}$ and $F_{1}$, for each force update, plotted as a function of the trajectory number. Values corresponding to the DD-HMC algorithm are shown to the left and the integration stepsizes for the two forces relate as $\Delta t_{2}: \Delta t_{1}=1: 6$. The values for MP-HMC are shown in the two right panels and the corresponding ratio of the integration steps is $\Delta t_{1}: \Delta t_{2}=1: 10$. The lattice size is $48 \times 24^{3}$ and $\kappa_{\text {sea }}=0.13625$. 
dynamical QCD simulations, with our implementation of a mass preconditioned HMC, including for the first time a locally deflated solver, which brings a significant speedup. We have confirmed that relatively large step size for small quark mass is achievable also with MP-HMC. Looking at the series of average and maximal forces of $F_{2}$ in both cases, it is indicative that the energy violations visible as spikes in $\Delta H$ are caused by the irregularities in the forces. This is easier to control with a continuous parameter, such as the preconditioning mass in mass preconditioning case, than with the HMC block size in DD-HMC which can only take few values in practical applications. The two algorithms have demonstrated comparable performance, which is in large owed to the usage of the same efficient deflated solver in both cases.

A future task is to extend this study to larger lattices with even lower quark masses. The MPHMC also leaves room for improvement. One could study the use of three or more pseudo-fermion fields and also tune the preconditioning masses more carefully than we did in the present results. In particular for smaller preconditioning masses, the use of the deflated solver also for $F_{1}$ might be advisable.

Besides being able to use larger numbers of CPUs, the approach with mass preconditioning allows for a much easier extension of the program package, such as introducing Schrödinger functional boundary conditions, as well as adding additional heavy and non-degenerate flavours.

\section{Acknowledgements}

We thank M. Hasenbusch, H. Simma, R. Sommer, F. Virotta and U. Wolff for useful and stimulating discussions and M. Lüscher for making his DD-HMC code publicly available. This work is supported by the German Science Foundation (DFG) under the grants GRK1504 "Mass, spectrum, symmetry" and SFB/TR9-03. Simulations were performed at HLRN in Berlin and Hannover.

\section{References}

[1] Martin Lüscher, Comput. Phys. Commun., 165:199-220, 2005.

[2] M. Hasenbusch, Phys. Lett., B519:177-182, 2001; M. Hasenbusch, K. Jansen, Nucl. Phys. B659 (2003) 299-320.

[3] M. Lüscher, JHEP 0707 (2007) 081.

[4] S. Schaefer, R. Sommer, F. Virotta, arXiv:1009.5228 [hep-lat].

[5] K. Jansen, A. Shindler, C. Urbach and U. Wenger, PoS LAT2005 (2006) 118.

[6] B. Leder, F. Knechtli, R. Sommer, PoS LAT2010 (2010) 233.

[7] L. Del Debbio, et al., JHEP 0702 (2007) 056; L. Del Debbio, et al. JHEP 0702 (2007) 082.

[8] R. C. Brower, T. Ivanenko, A. R. Levi and K. N. Orginos, Nucl. Phys. B 484 (1997) 353.

[9] M. Lüscher, Comput. Phys. Commun. 156 (2004) 209.

[10] M. Lüscher, JHEP 0712, 011 (2007). 